\title{
Non-Linear Reduced Round Attacks Against SHA-2 Hash family
}

\author{
Somitra Kumar Sanadhya ${ }^{\star}$ and Palash Sarkar \\ Applied Statistics Unit, \\ Indian Statistical Institute, \\ 203, B.T. Road, Kolkata, \\ India 700108. \\ somitra_r@isical.ac.in, palash@isical.ac.in
}

\begin{abstract}
Most of the attacks against (reduced) SHA-2 family in literature have used local collisions which are valid for linearized version of SHA-2 hash functions. Recently, at FSE '08, an attack against reduced round SHA-256 was presented by Nikolić and Biryukov which used a local collision which is valid for the actual SHA-256 function. It is a 9-step local collision which starts by introducing a modular difference of 1 in the two messages. It succeeds with probability roughly $1 / 3$. We build on the work of Nikolić and Biryukov and provide a generalized nonlinear local collision which accepts an arbitrary initial message difference. This local collision succeeds with probability 1. Using this local collision we present attacks against 18-step SHA256 and 18-step SHA-512 with arbitrary initial difference. Both of these attacks succeed with probability 1. We then present special cases of our local collision and show two different differential paths for attacking 20-step SHA-256 and 20-step SHA-512. One of these paths is the same as presented by Nikolić and Biryukov while the other one is a new differential path. Messages following both these differential paths can be found with probability 1 . This improves on the previous result where the success probability of 20-step attack was 1/3. Finally, we present two differential paths for 21-step collisions for SHA-256 and SHA-512, one of which is a new path. The success probability of these paths for SHA-256 is roughly $2^{-15}$ and $2^{-17}$ which improves on the 21-step attack having probability $2^{-19}$ reported earlier. We show examples of message pairs following all the presented differential paths for up to 21-step collisions in SHA-256. We also show first real examples of colliding message pairs for up to 20-step reduced SHA-512.
\end{abstract}

\section{Introduction}

Cryptanalysis of hash functions has been an area of intense interest to the research community since past decade and a half. Many hash functions were broken in this time, most notable among them are MD5 [12], SHA-0 [13] and theoretical break of SHA-1 [11]. This has directed the attention of the cryptology community to the SHA-2 family of hash functions.

Known Results for the SHA-2 Family: Gilbert and Handschuh (GH) [2] were the first to study local collisions in the SHA-2 family. They reported a 9-step local collision for linearized version of SHA256 and estimated the probability of the differential path to be $2^{-66}$. This probability estimate was later improved by Hawkes et al. [3]. Sanadhya and Sarkar [7] presented 16 new 9-step local collisions for SHA-2 family of hash functions. All these local collisions are also for the linearized version of SHA-256. In [8], an algorithm for generating 18-step SHA-256 collisions was developed using one of these local collisions and many colliding message pairs for 18-step SHA-256 were obtained. The message expansion of SHA-256 was studied by Mendel et al. [4], who reported a colliding message pair for 18-step SHA-256 which was recently corrected in [5]. They used the linearized local collision from [2] in their work. Mendel et al. [4] also improved the probability estimate of the Gilbert-Handschuh local collision to values similar to those obtained in [3].

Recently, Nikolić and Biryukov [6] presented a new local collision which uses modular differences instead of the XOR differences. Since this local collision is for the actual SHA-256 (and not its linearized version), its probability is much higher than the linearized local collisions presented earlier. For the first time in the literature, the authors in [6] worked directly with modular differences for SHA-256. Using this local collision they obtained 20-step and 21-step collisions for SHA-256 with probabilities 1/3 and $1 / 2^{19}$ respectively.

\footnotetext{
^ This author is supported by the Ministry of Information Technology, Govt. of India.
} 
Our Contributions: We build on the work of Nikolić and Biryukov [6] and present a generalized nonlinear local collision which accepts an arbitrary initial message difference. In [6], sufficient conditions for the differential path are determined and a particular local collision is obtained. We work with exact solutions of conditions imposed by the differential path and obtain general solutions of these conditions. Since we work with exact solutions of the conditions, our local collision is deterministic i.e. it holds with probability 1. Using this local collision, we obtain collisions for 18-step SHA-256 and 18-step SHA-512 with an arbitrary initial message difference. These attacks succeed with probability 1.

Then we show special instances of our generalized local collision which are suitable for finding collisions for 20-step SHA-256 and 20-step SHA-512. We present two such instances. One of these instances is a new local collision which can be realized in two different ways. The other one is the same as that presented by Nikolić and Biryukov for obtaining 20-step collision in [6]. However, unlike in [6], our 20-step attacks succeed with probability 1.

Finally, we use 20-step collisions to obtain 21-step collisions for SHA-256 as in [6]. There the probability for 21-step SHA-256 collisions is experimentally estimated to be about $2^{-19}$. We improve the efficiency of the probabilistic search used in this case and obtain 21-step collisions for SHA-256 with estimated experimental probability of $2^{-15}$. This is also the first time that actual collisions for SHA-512 reduced up to 20 steps are presented.

\section{Notation}

In this paper we use the following notation:

$-m_{i} \in\{0,1\}^{n}, W_{i} \in\{0,1\}^{n}, W_{i}^{\prime} \in\{0,1\}^{n}$ for any $i$. The word size $n$ is 32 for SHA-256 and 64 for SHA-512.

- The colliding message pair: $\left\{m_{0}, m_{1}, m_{2}, \ldots m_{15}\right\}$ and $\left\{m_{0}^{\prime}, m_{1}^{\prime}, m_{2}^{\prime}, \ldots m_{15}^{\prime}\right\}$.

- The expanded message pair: $\left\{W_{0}, W_{1}, W_{2}, \ldots W_{r-1}\right\}$ and $\left\{W_{0}^{\prime}, W_{1}^{\prime}, W_{2}^{\prime}, \ldots W_{r-1}^{\prime}\right\}$. The number of steps $r$ is 64 for SHA-256 and 80 for SHA-512.

- The internal registers for the two message pairs in step $i:\left\{a_{i}, \ldots, h_{i}\right\}$ and $\left\{a_{i}^{\prime}, \ldots, h_{i}^{\prime}\right\}$.

- $\operatorname{ROTR}^{k}(x)$ : Right rotation of an $n$-bit quantity $x$ by $k$ bits.

- $\operatorname{SHR}^{k}(x)$ : Right shift of an $n$-bit quantity $x$ by $k$ bits.

$-\oplus$ : bitwise XOR.

-+ : addition modulo $2^{n}$.

- - : subtraction modulo $2^{n}$.

- $\delta X=X^{\prime}-X$ where $\mathrm{X}$ is an $n$-bit quantity.

$-\delta \Sigma_{1}\left(e_{i}\right)=\Sigma_{1}\left(e_{i}^{\prime}\right)-\Sigma_{1}\left(e_{i}\right)$.

$-\delta \Sigma_{0}\left(a_{i}\right)=\Sigma_{0}\left(a_{i}^{\prime}\right)-\Sigma_{0}\left(a_{i}\right)$.

- $\delta f_{M A J}^{i}(x, y, z)$ : Output difference of the $f_{M A J}$ function in step $i$ when its inputs differ by $x, y$ and $z$. That is, $\delta f_{M A J}^{i}(x, y, z)=f_{M A J}\left(a_{i}+x, b_{i}+y, c_{i}+z\right)-f_{M A J}\left(a_{i}, b_{i}, c_{i}\right)$.

- $\delta f_{I F}^{i}(x, y, z)$ : Output difference of the $f_{I F}$ function in step $i$ when its inputs differ by $x, y$ and $z$. That is, $\delta f_{I F}^{i}(x, y, z)=f_{I F}\left(e_{i}+x, f_{i}+y, g_{i}+z\right)-f_{I F}\left(e_{i}, f_{i}, g_{i}\right)$.

\section{The SHA-2 Hash Family}

The newest members of SHA family of hash functions were standardized by US NIST in 2002 [10]. There are 2 differently designed functions in this standard: the SHA-256 and SHA-512. In addition, the standard also specifies their truncated version: SHA-224 and SHA-384. The number in the name of the hash function refers to the length of message digest produced by that function. Next we describe SHA-256 and SHA-512 in detail.

The round function of SHA-2 hash family is shown in Figure 1. Eight registers are used in the evaluation of SHA-2. The initial value in the registers is specified by an $8 \times n$ bit IV, $n=32$ for SHA-256 
Fig. 1. Round function of SHA-2 hash family

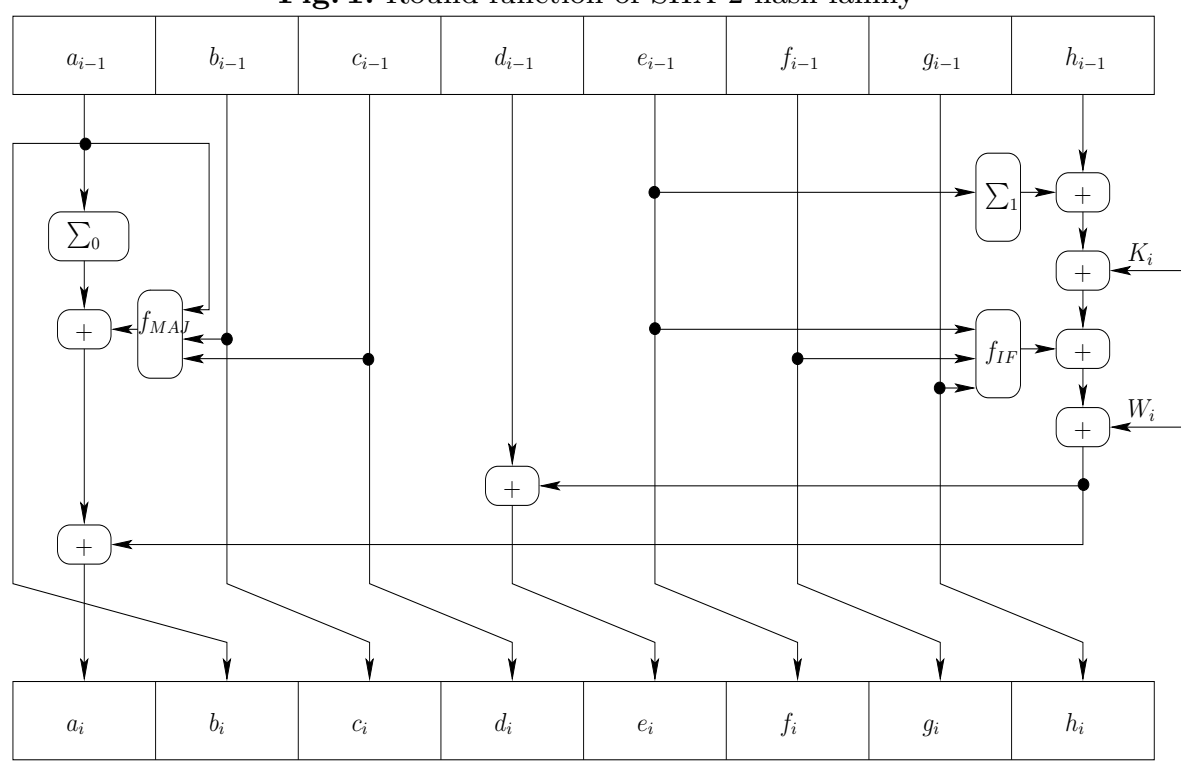

and 64 for SHA-512. In Step $i$, the 8 registers are updated from $\left(a_{i-1}, b_{i-1}, c_{i-1}, d_{i-1}, e_{i-1}, f_{i-1}, g_{i-1}\right.$, $\left.h_{i-1}\right)$ to $\left(a_{i}, b_{i}, c_{i}, d_{i}, e_{i}, f_{i}, g_{i}, h_{i}\right)$ according to the following equations:

$$
\left.\begin{array}{rl}
a_{i}= & \Sigma_{0}\left(a_{i-1}\right)+f_{M A J}\left(a_{i-1}, b_{i-1}, c_{i-1}\right)+\Sigma_{1}\left(e_{i-1}\right) \\
& +f_{I F}\left(e_{i-1}, f_{i-1}, g_{i-1}\right)+h_{i-1}+K_{i}+W_{i} \\
b_{i}= & a_{i-1} \\
c_{i}= & b_{i-1} \\
d_{i}= & c_{i-1} \\
e_{i}= & d_{i-1}+\Sigma_{1}\left(e_{i-1}\right)+f_{I F}\left(e_{i-1}, f_{i-1}, g_{i-1}\right) \\
& +h_{i-1}+K_{i}+W_{i} \\
f_{i}= & e_{i-1} \\
g_{i}= & f_{i-1} \\
h_{i}= & g_{i-1}
\end{array}\right\}
$$

The $f_{I F}$ and the $f_{M A J}$ are three variable boolean functions defined as:

$$
\begin{aligned}
& f_{I F}(x, y, z)=(x \wedge y) \oplus(\neg x \wedge z), \\
& f_{M A J}(x, y, z)=(x \wedge y) \oplus(y \wedge z) \oplus(z \wedge x) .
\end{aligned}
$$

For SHA-256, the functions $\Sigma_{0}$ and $\Sigma_{1}$ are defined as:

$$
\begin{aligned}
& \Sigma_{0}(x)=\operatorname{ROTR}^{2}(x) \oplus \operatorname{ROTR}^{13}(x) \oplus \operatorname{ROTR}^{22}(x), \\
& \Sigma_{1}(x)=\operatorname{ROTR}^{6}(x) \oplus \operatorname{ROTR}^{11}(x) \oplus \operatorname{ROTR}^{25}(x) .
\end{aligned}
$$

For SHA-512, the corresponding functions are:

$$
\begin{aligned}
& \Sigma_{0}(x)=\operatorname{ROTR}^{28}(x) \oplus \operatorname{ROTR}^{34}(x) \oplus \operatorname{ROTR}^{39}(x), \\
& \Sigma_{1}(x)=\operatorname{ROT}^{14}(x) \oplus \operatorname{ROT} R^{18}(x) \oplus \operatorname{ROTR}^{41}(x) .
\end{aligned}
$$

Round $i$ uses a $n$-bit word $W_{i}$ which is derived from the message and a constant word $K_{i}$. There are 64 steps in SHA-256 and 80 in SHA-512. The hash function operates on a 512-bit (resp. 1024-bit) 
message specified as 16 words of 32 (resp. 64) bits for SHA-256 (resp. SHA-512). Given the message words $m_{0}, m_{1}, \ldots m_{15}$, the $W_{i}$ 's are computed using the equation:

$$
W_{i}= \begin{cases}m_{i} & \text { for } 0 \leq i \leq 15 \\ \sigma_{1}\left(W_{i-2}\right)+W_{i-7}+\sigma_{0}\left(W_{i-15}\right)+W_{i-16} & \text { for } 16 \leq i \leq 63(\text { or } 80)\end{cases}
$$

For SHA-256, the functions $\sigma_{0}$ and $\sigma_{1}$ are defined as:

$$
\begin{aligned}
& \sigma_{0}(x)=\operatorname{ROTR}^{7}(x) \oplus \operatorname{ROTR}^{18}(x) \oplus \operatorname{SHR}^{3}(x), \\
& \sigma_{1}(x)=\operatorname{ROTR}^{17}(x) \oplus \operatorname{ROTR} R^{19}(x) \oplus \operatorname{SHR}^{10}(x) .
\end{aligned}
$$

And for SHA-512, they are defined as:

$$
\begin{aligned}
& \sigma_{0}(x)=\operatorname{ROTR}^{1}(x) \oplus \operatorname{ROTR}^{8}(x) \oplus \operatorname{SHR}^{7}(x), \\
& \sigma_{1}(x)=\operatorname{ROTR}^{19}(x) \oplus \operatorname{ROTR}^{61}(x) \oplus \operatorname{SHR}^{6}(x) .
\end{aligned}
$$

The IV $=\left(a_{-1}, b_{-1}, c_{-1}, d_{-1}, e_{-1}, f_{-1}, g_{-1}, h_{-1}\right)$ is defined as (0x6a09e667, 0xbb67ae85, 0x3c6ef372, 0xa54ff53a, 0x510e527f, 0x9b05688c, 0x1f83d9ab, 0x5be0cd19) for SHA-256. Different IV values are defined for SHA-224, SHA-384 and SHA-512. For details, see [10].

The output hash value of a one block (512-bit for SHA-256 and 1024-bit for SHA-512) message is obtained by chaining the IV with the register values at the end of the final round as per the MerkleDamgård construction. A similar strategy is used for multi-block messages, where the IV for next block is taken as the hash output of the previous block.

\section{Collision Attacks Against the SHA-2 Hash Family}

The aim of collision attacks against hash functions is to obtain two different messages which produce the same digest under that hash function. The hash functions use one word of the message in each step and process the message for multiple steps. Typically, an attacker introduces a small difference in one word of the message. Using the terminology from [1], this initial difference is called the "perturbation message difference". Next few message words are chosen to differ in such a manner that all the introduced differences cancel themselves with high probability. These later message word differences are called "correction differences".

Not all the message words used in different steps of the hash function are freely available to the attacker. Most of the hash designs have 16 words of freedom which is available in the first 16 steps of hash evaluation. Rest of the message words are computed on the basis of the first 16 words using "message expansion".

A "local collision" is a collision producing differential path (and the message differences required for this path) spanning a small number of steps in which the message expansion in not considered. Gilbert and Handschuh reported the first local collision for SHA-256 [2] using XOR differences in the message words. Sanadhya and Sarkar [7] reported 16 other local collisions, all of which used XOR differences in the message words. Very recently, Nikolić and Biryukov [6] showed a different type of local collision, which used modular differences in the message words. The XOR difference based local collisions have linear property, i.e. any number of them can be freely superimposed, whereas the modular difference based local collision can not be superimposed freely. For this reason, we refer to them as "linear local collisions" and "nonlinear local collisions" respectively.

We present our new nonlinear local collision next.

\section{A General Class Of Nonlinear Local Collisions}

Table 1 shows the general structure of a 9-step local collision for SHA-2 family. The perturbation message difference is taken to be $x$ and other message differences are later computed. In Table 1, the registers $\left(a_{i-1}, \ldots, h_{i-1}\right)$ and $W_{i}$ are inputs to Step $i$ of the hash evaluation and this step outputs the registers $\left(a_{i}, \ldots, h_{i}\right)$. 
Table 1. A 9-step nonlinear local collision for SHA-256.

\begin{tabular}{|c|c|c|c|c|c|c|c|c|c|}
\hline Step $i$ & $\delta W_{i}$ & $\delta a_{i}$ & $\delta b_{i}$ & $\delta c_{i}$ & $\delta d_{i}$ & $\delta e_{i}$ & $\delta f_{i}$ & $\delta g_{i}$ & $\delta h_{i}$ \\
\hline$i-1$ & 0 & 0 & 0 & 0 & 0 & 0 & 0 & 0 & 0 \\
\hline$i$ & $x$ & $x$ & 0 & 0 & 0 & $x$ & 0 & 0 & 0 \\
\hline$i+1$ & $\delta W_{i+1}$ & 0 & $x$ & 0 & 0 & $y$ & $x$ & 0 & 0 \\
\hline$i+2$ & $\delta W_{i+2}$ & 0 & 0 & $x$ & 0 & $z$ & $y$ & $x$ & 0 \\
\hline$i+3$ & $\delta W_{i+3}$ & 0 & 0 & 0 & $x$ & 0 & $z$ & $y$ & $x$ \\
\hline$i+4$ & $\delta W_{i+4}$ & 0 & 0 & 0 & 0 & $x$ & 0 & $z$ & $y$ \\
\hline$i+5$ & $\delta W_{i+5}$ & 0 & 0 & 0 & 0 & 0 & $x$ & 0 & $z$ \\
\hline$i+6$ & $\delta W_{i+6}$ & 0 & 0 & 0 & 0 & 0 & 0 & $x$ & 0 \\
\hline$i+7$ & $\delta W_{i+7}$ & 0 & 0 & 0 & 0 & 0 & 0 & 0 & $x$ \\
\hline$i+8$ & $\delta W_{i+8}$ & 0 & 0 & 0 & 0 & 0 & 0 & 0 & 0 \\
\hline
\end{tabular}

\subsection{Message Word Differences for Table 1}

In Step $i$ of SHA-2, only the registers $a_{i}$ and $e_{i}$ are computed. Rest of the registers are copies of the old ones. Therefore we focus on these two register evaluations only. From (1), we get:

$$
\begin{aligned}
\delta e_{i}=\delta \Sigma_{1}\left(e_{i-1}\right)+\delta f_{I F}\left(\delta e_{i-1}, \delta f_{i-1}, \delta g_{i-1}\right)+\delta d_{i-1}+\delta h_{i-1}+\delta W_{i} \\
\delta a_{i}=\delta \Sigma_{0}\left(a_{i-1}\right)+\delta f_{M A J}\left(\delta a_{i-1}, \delta b_{i-1}, \delta c_{i-1}\right)+\delta \Sigma_{1}\left(e_{i-1}\right)+ \\
=\delta f_{I F}\left(\delta e_{i-1}, \delta f_{i-1}, \delta g_{i-1}\right)+\delta h_{i-1}+\delta W_{i} \\
=\delta \Sigma_{0}\left(a_{i-1}\right)+\delta f_{M A J}\left(\delta a_{i-1}, \delta b_{i-1}, \delta c_{i-1}\right)+\delta e_{i}-\delta d_{i-1}
\end{aligned}
$$

We now try to satisfy the restriction imposed by the differential path of Table 1 by defining suitable difference of the message words in various steps.

Step i : If $\delta W_{i}=x$, then this difference will propagate to both the registers $a_{i}$ and $e_{i}$.

Step (i+1) : At this step $a_{i}^{\prime}-a_{i}=e_{i}^{\prime}-e_{i}=x$. We want $\delta a_{i+1}=0$ and $\delta e_{i+1}=y$. From (3) and (2), we get:

$$
\begin{aligned}
& \delta a_{i+1}=0=\delta \Sigma_{0}\left(a_{i}\right)+\delta f_{M A J}^{i}(x, 0,0)+\delta \Sigma_{1}\left(e_{i}\right)+\delta f_{I F}^{i}(x, 0,0)+\delta W_{i+1} \\
& \delta e_{i+1}=y=\delta \Sigma_{1}\left(e_{i}\right)+\delta f_{I F}^{i}(x, 0,0)+\delta W_{i+1}
\end{aligned}
$$

The exact solution of the equations above is:

$$
\begin{gathered}
y=-\delta \Sigma_{0}\left(a_{i}\right)-\delta f_{M A J}^{i}(x, 0,0), \\
\delta W_{i+1}=y-\delta f_{I F}^{i}(x, 0,0)-\delta \Sigma_{1}\left(e_{i}\right) .
\end{gathered}
$$

Step $(\mathbf{i}+2):$ At this step $b_{i+1}^{\prime}-b_{i+1}=f_{i+1}^{\prime}-f_{i+1}=x$ and $e_{i+1}^{\prime}-e_{i+1}=y$. We want $\delta a_{i+2}=0$ and $\delta e_{i+2}=z$. From (3) and (2), we get:

$$
\begin{aligned}
& \delta a_{i+2}=0=\delta f_{M A J}^{i+1}(0, x, 0)+\delta \Sigma_{1}\left(e_{i+1}\right)+\delta f_{I F}^{i+1}(y, x, 0)+\delta W_{i+2}, \\
& \delta e_{i+2}=z=\delta \Sigma_{1}\left(e_{i+1}\right)+\delta f_{I F}^{i+1}(y, x, 0)+\delta W_{i+2} .
\end{aligned}
$$

The conditions above translate to:

$$
\begin{gathered}
z=-\delta f_{M A J}^{i+1}(0, x, 0) \\
\delta W_{i+2}=z-\delta f_{I F}^{i+1}(y, x, 0)-\delta \Sigma_{1}\left(e_{i+1}\right) .
\end{gathered}
$$


Step $(\mathbf{i}+3):$ At this $\operatorname{step} c_{i+2}^{\prime}-c_{i+2}=g_{i+2}^{\prime}-g_{i+2}=x, e_{i+2}^{\prime}-e_{i+2}=z$ and $f_{i+2}^{\prime}-f_{i+2}=y$. We want $\delta a_{i+3}=0$ and $\delta e_{i+3}=0$. From (3) and (2), we get:

$$
\begin{aligned}
& \delta a_{i+3}=0=\delta f_{M A J}^{i+2}(0,0, x)+\delta \Sigma_{1}\left(e_{i+2}\right)+\delta f_{I F}^{i+2}(z, y, x)+\delta W_{i+3}, \\
& \delta e_{i+3}=0=\delta \Sigma_{1}\left(e_{i+2}\right)+\delta f_{I F}^{i+2}(z, y, x)+\delta W_{i+3} .
\end{aligned}
$$

The conditions above translate to:

$$
\begin{gathered}
\delta f_{M A J}^{i+2}(0,0, x)=0 \\
\delta W_{i+3}=-\delta f_{I F}^{i+2}(z, y, x)-\delta \Sigma_{1}\left(e_{i+2}\right) .
\end{gathered}
$$

Step $(\mathbf{i}+4)$ : At this step $d_{i+3}^{\prime}-d_{i+3}=h_{i+3}^{\prime}-h_{i+3}=x, f_{i+3}^{\prime}-f_{i+3}=z$ and $g_{i+3}^{\prime}-g_{i+3}=y$. We want $\delta a_{i+4}=0$ and $\delta e_{i+4}=x$. From (3) and (2), we get:

$$
\begin{aligned}
& \delta a_{i+4}=0=\delta f_{I F}^{i+3}(0, z, y)+x+\delta W_{i+4}, \\
& \delta e_{i+4}=x=\delta f_{I F}^{i+3}(0, z, y)+x+x+\delta W_{i+4} .
\end{aligned}
$$

The conditions above translate to:

$$
\delta W_{i+4}=-x-\delta f_{I F}^{i+3}(0, z, y) .
$$

Step $(\mathbf{i}+5)$ : At this step $e_{i+4}^{\prime}-e_{i+4}=x, g_{i+4}^{\prime}-g_{i+4}=z$ and $h_{i+4}^{\prime}-h_{i+4}=y$. We want $\delta a_{i+5}=$ $\delta e_{i+5}=0$. From (3) and (2), we get:

$$
\begin{aligned}
& \delta a_{i+5}=0=\delta \Sigma_{1}\left(e_{i+4}\right)+\delta f_{I F}^{i+4}(x, 0, z)+y+\delta W_{i+5}, \\
& \delta e_{i+5}=0=\delta \Sigma_{1}\left(e_{i+4}\right)+\delta f_{I F}^{i+4}(x, 0, z)+y+\delta W_{i+5} .
\end{aligned}
$$

The conditions above translate to:

$$
\delta W_{i+5}=-y-\delta f_{I F}^{i+4}(x, 0, z)-\delta \Sigma_{1}\left(e_{i+4}\right) .
$$

Step (i+6) : At this step $f_{i+5}^{\prime}-f_{i+5}=x$ and $h_{i+5}^{\prime}-h_{i+5}=z$. We want $\delta a_{i+6}=\delta e_{i+6}=0$. From (3) and (2), we get:

$$
\begin{aligned}
& \delta a_{i+6}=0=\delta f_{I F}^{i+5}(0, x, 0)+z+\delta W_{i+6}, \\
& \delta e_{i+6}=0=\delta f_{I F}^{i+5}(0, x, 0)+z+\delta W_{i+6} .
\end{aligned}
$$

The conditions above translate to:

$$
\delta W_{i+6}=-z-\delta f_{I F}^{i+5}(0, x, 0) .
$$

Step $(\mathbf{i}+7):$ At this step $g_{i+6}^{\prime}-g_{i+6}=x$. We want $\delta a_{i+7}=\delta e_{i+7}=0$. From (3) and (2), we get:

$$
\begin{aligned}
& \delta a_{i+7}=0=\delta f_{I F}^{i+6}(0,0, x)+\delta W_{i+7}, \\
& \delta e_{i+7}=0=\delta f_{I F}^{i+6}(0,0, x)+\delta W_{i+7} .
\end{aligned}
$$

The conditions above translate to:

$$
\delta W_{i+7}=-\delta f_{I F}^{i+6}(0,0, x) .
$$

Step (i+8) : At this step $h_{i+7}^{\prime}-h_{i+7}=x$. We want $\delta a_{i+8}=\delta e_{i+8}=0$. This will happen as desired if we have:

$$
\delta W_{i+8}=-x
$$




\subsection{Solution of Equations}

To find a local collision, we need message pairs which will satisfy (4) to (14). Out of these, only (8) puts restrictions on the message pair. Rest of the equations merely define the correction message differences. For clarity, we reproduce the condition here.

$$
\delta f_{M A J}^{i+2}(0,0, x)=0 .
$$

Next we explain how to satisfy this condition easily. This is based on the technique in [6]. The $f_{M A J}$ function has registers $(a, b, c)$ as inputs. The necessary condition for the two different inputs to the $f_{M A J}$ to not propagate the difference in the output is that :

- Registers $a_{i+2}$ and $b_{i+2}$ must have same value at those bit positions where registers $c_{i+2}^{\prime}$ and $c_{i+2}$ differ.

Note that $b_{i+2}=a_{i+1}$. Although the condition above requires us to ensure equality of bit patterns in the two registers only at some places, we can strengthen this condition a little and try to make these register values exactly equal. Thus, we need to satisfy $a_{i+2}=a_{i+1}$.

Note that we have put no restriction on the message words themselves in solving earlier equations. The only restrictions are on the "difference" of messages. To ensure the equality of the registers as desired, we can now put some conditions on the actual message word $W_{i+2}$. When the $(i+2)^{t h}$ step of the hash evaluation is executed, the registers $\left\{a_{i+1}, \ldots, h_{i+1}\right\}$ will already be available. So we can choose $W_{i+2}$ such that it produces a value in register $a_{i+2}$ which is equal to the already known value $a_{i+1}$. This requires solving the following equations simultaneously:

$$
\begin{aligned}
& a_{i+2}=\Sigma_{0}\left(a_{i+1}\right)+f_{M A J}\left(a_{i+1}, b_{i+1}, c_{i+1}\right)+\Sigma_{1}\left(e_{i+1}\right)+f_{I F}\left(e_{i+1}, f_{i+1}, g_{i+1}\right)+h_{i+1}+K_{i+2}+W_{i+2}, \\
& a_{i+2}=a_{i+1} .
\end{aligned}
$$

Hence, we choose $W_{i+2}$ such that:

$$
W_{i+2}=a_{i+1}-\Sigma_{0}\left(a_{i+1}\right)-f_{M A J}\left(a_{i+1}, b_{i+1}, c_{i+1}\right)-\Sigma_{1}\left(e_{i+1}\right)-f_{I F}\left(e_{i+1}, f_{i+1}, g_{i+1}\right)-h_{i+1}-K_{i+2} .
$$

\subsection{Obtaining a Local Collision}

To obtain the 9-step local collision as in Table 1, we first select the perturbation message difference $\delta W_{i}$ as a randomly generated 32-bit (or 64-bit) quantity $x$. The differences $\delta W_{j}$ for $j \in\{(i+1), \ldots,(i+8)\}$ are defined by (5), (7), (9), (10), (11), (12), (13) and (14). In addition, as discussed in the last section, we choose $W_{i+2}$ such that (15) is satisfied. Rest of the message words could be any randomly chosen 32-bit (or 64-bit) words. This local collision holds with probability 1, since all the steps are deterministic and feasible.

\section{Extending a Single Local Collision to Obtain 18-Step Collisions}

In this section we explain how to obtain 18-step collisions using the local collision shown in this paper. We discuss three different types of differential paths depending on the value of the differential $z$ used in $\delta e_{i+2}$ to $\delta h_{i+5}$ in Table 1 .

For all the different cases that we describe next, we choose to span the 9-step local collision from Step 3 to Step 11. The message differentials $\delta W_{i}$ for $i \in\{3,4, \ldots, 11\}$ are defined by the local collision. We use a single local collision, which implies that all the other free message words are equal. That is, $\delta W_{i}=0$ for $i \in\{0,1,2,12,13,14,15\}$.

First two steps of message expansion of SHA-2 define the message words $W_{16}$ and $W_{17}$ as follows:

$$
\begin{aligned}
& W_{16}=\sigma_{1}\left(W_{14}\right)+W_{9}+\sigma_{0}\left(W_{1}\right)+W_{0} \\
& W_{17}=\sigma_{1}\left(W_{15}\right)+W_{10}+\sigma_{0}\left(W_{2}\right)+W_{1}
\end{aligned}
$$


From these two equations, it is clear that if $\delta W_{9}=\delta W_{10}=0$ then the two expanded message words will be equal for Steps 17 and 18. This will result in an 18-step collision for SHA-2. Note that $\delta W_{9}$ and $\delta W_{10}$ correspond to Steps 7 and 8 of the local collision used. Hence our target is to get differentials of the message in these two steps to vanish.

\subsection{When $z=0$ in the Local Collision}

In this case the local collision looks similar to the one given in [6]. But note that that our local collision accepts any random message difference $x$, whereas in [6] the specific value $x=1$ is used.

As explained above, we need to ensure that (12) and (13) give zero differences. In addition we also need $z=0$ from (6).

To get $z=0$, we need to have $\delta f_{M A J}^{4}(0, x, 0)=0$. Similar to the methods used in Section 5.2, we can ensure this by the sufficient condition $a_{4}=c_{4}$ (i.e. $a_{4}=a_{2}$ ) which can be deterministically satisfied by suitable choice of $W_{4}$.

Next we need two consecutive message differences zero at Steps 10 and 11 of the differential path. Equation 12 corresponding to Step 10 gives zero difference if $\delta f_{I F}^{8}(0, x, 0)=0$. This can be deterministically satisfied by choosing $W_{8}$ such that $e_{8}=0$. In this case, $f_{I F}$ selects its third argument which does not have any difference. Similarly (13) can be satisfied by choosing $W_{9}$ such that $\delta f_{I F}^{9}(0,0, x)=0$ i.e. this time we need $e_{9}=-1$. Thus we can deterministically obtain 18-step collisions for SHA-2 for any random initial perturbation $x$. Message pairs colliding for 18-step SHA-256 and for 18-step SHA-512 with initial perturbation selected randomly are given in Section A.

\subsection{When $z \neq 0$ in the Local Collision}

As before, we need that (12) and (13) give zero difference in message words. Stating these equations explicitly, we require that:

$$
\begin{gathered}
-z=f_{I F}\left(e_{8}, f_{8}+x, g_{8}\right)-f_{I F}\left(e_{8}, f_{8}, g_{8}\right), \\
0=f_{I F}\left(e_{9}, f_{9}, g_{9}+x\right)-f_{I F}\left(e_{9}, f_{9}, g_{9}\right) .
\end{gathered}
$$

Equation 17 is easy to satisfy by selecting $W_{9}$ such that $e_{9}=-1$. Then $f_{I F}$ selects its second argument which does not have any difference. However, (16) is not easily satisfied this time. This equation is easy to solve only for special $z$ values of the type $z=0$ or $z= \pm x$. To have an 18-step collision, we need $z$ to take these special values. We discuss the two non-zero cases for $z$ separately.

When $z=-x$ : The value $z$ gets defined by (6). So we need to handle this equation, which states that:

$$
z=-\delta f_{M A J}^{4}(0, x, 0) .
$$

This puts restrictions on the values of the registers $\left\{a_{4}, b_{4}, c_{4}\right\}$ and perturbation difference $x$ such that the following condition holds:

$$
f_{M A J}\left(a_{4}, b_{4}+x, c_{4}\right)-f_{M A J}\left(a_{4}, b_{4}, c_{4}\right)=x .
$$

Left hand side of (18) can be thought of as a function which accepts 4 words of input and returns 1 word of output. Clearly, there are many solutions to this equation. One solution to this equation is $a_{4}=-1, c_{4}=0$ with $b_{4}$ being any arbitrary value. For any $x$, this will be a solution to (18). [This solution was suggested by an anonymous reviewer of ACISP 2008.] Alternately, random search of the 4 word space can be made which also quickly gives solutions for (18). The cost of finding random solutions to this equation is also negligible.

Once some values for $\left(a_{4}, b_{4}, c_{4}\right)=\left(a_{4}, a_{3}, a_{2}\right)$ and $x$ which satisfy (18) have been selected, we need to have these register values in the differential path at the appropriate step. This can be done by choosing 
$W_{4}, W_{3}$ and $W_{2}$ appropriately. Let the selected values of $\left(a_{4}, a_{3}, a_{2}\right)$ be $(\alpha, \beta, \gamma)$. Then the message words should be chosen to satisfy the following equations:

$$
\begin{gathered}
W_{2}=\gamma-\Sigma_{0}\left(a_{1}\right)-f_{M A J}\left(a_{1}, b_{1}, c_{1}\right)-\Sigma_{1}\left(e_{1}\right)-f_{I F}\left(e_{1}, f_{1}, g_{1}\right)-h_{1}-K_{2}, \\
W_{3}=\beta-\Sigma_{0}(\gamma)-f_{M A J}\left(\gamma, b_{2}, c_{2}\right)-\Sigma_{1}\left(e_{2}\right)-f_{I F}\left(e_{2}, f_{2}, g_{2}\right)-h_{2}-K_{3}, \\
W_{4}=\alpha-\Sigma_{0}(\beta)-f_{M A J}\left(\beta, \gamma, c_{3}\right)-\Sigma_{1}\left(e_{3}\right)-f_{I F}\left(e_{3}, f_{3}, g_{3}\right)-h_{3}-K_{4} .
\end{gathered}
$$

We also need to satisfy (16). This is easily handled by having $e_{8}=-1$ so that $f_{I F}$ selects its middle argument and propagates the difference $x$. This can be done by choosing $W_{8}$ appropriately.

To summarize, we start a local collision spanning Steps 3 to 11 and choose some values of $\left(a_{4}, a_{3}, a_{2}\right)$ and $x$ such that (18) is satisfied. The differences in messages words $\delta W_{i}$ for $i \in\{3,4, \ldots 11\}$ are defined by the local collision. In addition, we select message words $W_{2}, W_{3}$ and $W_{4}$ by solving (19), (20) and (21). The local collision also requires us to choose $W_{5}$ in a particular manner (as explained in Section 5.2). Finally, we need to choose $W_{8}$ and $W_{9}$ so as to ensure that $e_{8}=e_{9}=-1$. Rest of the message words can be selected randomly. Note that we must first select $W_{0}$ and $W_{1}$ and then only can we solve for $W_{2}, W_{3}$ and $W_{4}$ etc. Further, the only cost involved in obtaining such 18-step collisions is in selecting suitable values of $\left(a_{4}, b_{4}, c_{4}\right)$ and $x$. The 18-step collision, which is obtained after any solution of (18) is chosen, holds with probability 1.

When $z=x$ : This time the majority condition takes the form:

$$
f_{M A J}\left(a_{4}, b_{4}+x, c_{4}\right)-f_{M A J}\left(a_{4}, b_{4}, c_{4}\right)=-x .
$$

There are many solutions to this equation as well. In particular, one subset of solutions is given by the following choice of the variables: $a_{4}=b_{4}=p$ and $c_{4}=p+x$ where $p$ is any arbitrary 32-bit quantity and $x=2^{31}$. This solution works because $2^{31}=-2^{31}$ in modulo $2^{32}$ arithmetic. The SHA-512 case is similar where we can use $2^{63}$ in place of $2^{31}$. The cost for finding random 32-bit solutions for the above equation is experimentally found to be about $2^{24}$. This means that finding a random solution for SHA-256 takes a few seconds on an ordinary PC. Few such solutions for 32-bit words are listed in Table 2.

Table 2. Example values of register $(a, b, c)$ and $x$ such that $f_{M A J}(a, b+x, c)-f_{M A J}(a, b, c)=-x$. Registers $a$ and $c$ can also be exchanged due to the symmetry of $f_{M A J}$.

\begin{tabular}{|c|l|l|l|l|}
\hline No. & $a$ & $b$ & $c$ & $x$ \\
\hline 1 & 0 & 0 & 80000000 & 80000000 \\
\hline 2 & $44070 d 26$ & 9f85286b & $823480 \mathrm{~b} 1$ & $7 \mathrm{ffdfffc}$ \\
\hline 3 & $1 \mathrm{~b} 1704 \mathrm{f} 1$ & $511209 \mathrm{a} 2$ & $\mathrm{f} 504556 \mathrm{a}$ & 00000100 \\
\hline 4 & fcbeab96 & a56c2117 & 0f94f865 & fe27f002 \\
\hline 5 & a4cffbbd & 8266ace3 & 392a62f6 & fffffffa \\
\hline
\end{tabular}

In this case, (12) and (13) are:

$$
\begin{aligned}
& \delta W_{9}=-x-\delta f_{I F}^{8}(0, x, 0) \\
& \delta W_{10}=-\delta f_{I F}^{9}(0,0, x)
\end{aligned}
$$

The right hand side of the second equation above can be made zero by choosing $e_{9}=-1$ so that the $f_{I F}$ function chooses its middle argument. This can be achieved by suitably choosing $W_{9}$. We use random choices of words to obtain $\delta W_{9}=0$. The complexity of this step is directly related to the hamming weight of $x$. For a 1-bit $x$ the probability of satisfying this step is about $1 / 2$ to $1 / 2^{3}$. For 
20-bit $x$ this equation gets satisfied with probability about $1 / 2^{8}$ to $1 / 2^{20}$. This cost is equivalent to a fraction of a second on an ordinary PC.

To summarize, we start a local collision spanning steps 3 to 11 and choose some values of $\left(a_{4}, a_{3}, a_{2}\right)$ and $x$ such that (22) is satisfied. The differences in messages words $\delta W_{i}$ for $i \in\{3,4, \ldots 11\}$ are defined by the local collision. In addition, we select message words $W_{2}, W_{3}$ and $W_{4}$ by solving (19), (20) and (21). The word $W_{8}$ is selected as explained above. Besides, $W_{5}$ is selected in the same way as in Section 5.2. Rest of the message words can be selected randomly.

There are two costs involved in obtaining such 18-step collisions: (1) Selecting suitable values of $\left(a_{4}, b_{4}, c_{4}\right)$ and $x$ satisfying (22), and (2) Satisfying $\delta W_{9}=0$. The first condition can be always satisfied by choosing suitable pre-computed values. The only probability for such 18-step collisions comes from the satisfaction of the second condition.

Message pairs colliding for 18-step SHA-256 and 18-step SHA-512 following this differential path (for both the cases $z=x$ and $z=-x$ ) are shown in Section A.

\section{Extending a Single Local Collision to Obtain 20-Step Collisions}

We follow the technique used in [6] to obtain 20-step collisions for SHA-256. This time we need to handle first 4 steps of message expansion. These steps are:

$$
\begin{aligned}
& W_{16}=\sigma_{1}\left(W_{14}\right)+W_{9}+\sigma_{0}\left(W_{1}\right)+W_{0} \\
& W_{17}=\sigma_{1}\left(W_{15}\right)+W_{10}+\sigma_{0}\left(W_{2}\right)+W_{1} \\
& W_{18}=\sigma_{1}\left(W_{16}\right)+W_{11}+\sigma_{0}\left(W_{3}\right)+W_{2} \\
& W_{19}=\sigma_{1}\left(W_{17}\right)+W_{12}+\sigma_{0}\left(W_{4}\right)+W_{3}
\end{aligned}
$$

If a single local collision spanning from Step 5 to Step 13 is used and all other messages outside the scope of this local collision are taken to have zero differentials, then $\delta W_{i}=0$ for $i \in\{0,1,2,3,4,14,15\}$. This implies that if we can have $\delta W_{9}=\delta W_{10}=\delta W_{11}=\delta W_{12}=0$, then the differentials of the first 4 expanded message words will be zero. In this case the message expansion will not play a role and we will be able to extend a single local collision to 20 steps.

The local collision presented in [6] is such that the message differentials at steps $i+4$ to $i+7$ are zero for it ( $i=5$ is the starting step of the local collision). Hence it can be used to obtain 20-step collisions directly. The local collision we presented is more general but does not necessarily have 4 consecutive message differentials equal to zero. Now we find particular instances of our local collision such that we have zero differentials as desired. This time we work with sufficient conditions as in [6].

To obtain the 4 consecutive zero differentials in the local collision, we need to have differentials generated by (10), (11), (12) and (13) (corresponding to Steps 9, 10, 11 and 12 of the differential path) to be equal to zero. We next discuss the conditions put by these equations. We also need to control the values of $y$ and $z$ by (4) and (6). As in [6], we start the local collision by choosing $x=1$.

Equation 4: This equation contains the term $\delta \Sigma_{0}\left(a_{5}\right)=\Sigma_{0}\left(a_{5}^{\prime}\right)-\Sigma_{0}\left(a_{5}\right)$. From the differential path we know that $\delta a_{5}=a_{5}^{\prime}-a_{5}=x$. Differential behavior of the non-linear function $\Sigma_{0}$ is difficult to analyze. To make it tractable, we choose $\delta \Sigma_{0}\left(a_{5}\right)=x=1$. For this case, the only solutions are $a_{5}=-1=0 \times \operatorname{xfffffff}$ and $a_{5}^{\prime}=0$. We also put restriction that the $f_{M A J}$ term doesn't propagate any difference. This condition $f_{M A J}^{5}(x, 0,0)=0$ implies $b_{5}=c_{5}$, i.e. $a_{4}=a_{3}$. Conditions on $a_{4}$ and $a_{5}$ registers can be deterministically satisfied by choosing $W_{4}$ and $W_{5}$ suitably. By the choices made above, this equation gives $y=-1$.

Equation 11: This equation contains the term $\delta \Sigma_{1}\left(e_{9}\right)=\Sigma_{1}\left(e_{9}^{\prime}\right)-\Sigma_{1}\left(e_{9}\right)$. From the differential path we know that $\delta e_{9}=e_{9}^{\prime}-e_{9}=x$. Differential behaviour of the non-linear function $\Sigma_{1}$ is difficult to analyze. Similar to the previous equation, we choose $\delta \Sigma_{1}\left(e_{9}\right)=x=1$. Once again, the only solutions 
are $e_{9}=-1=0 x f f f f f f f$ and $e_{9}^{\prime}=0$. This condition can be deterministically satisfied by choosing $W_{9}$ suitably. Finally, we wish to make the following difference zero:

$$
\begin{aligned}
\delta W_{10} & =-y-\delta f_{I F}^{9}(x, 0, z)-\delta \Sigma_{1}\left(e_{9}\right) \\
& =-(-1)-\left(f_{I F}\left(e_{9}+1, e_{8}, e_{7}+z\right)-f_{I F}\left(e_{9}, e_{8}, e_{7}\right)\right)-\left(\Sigma\left(e_{9}+1\right)-\Sigma_{1}\left(e_{9}\right)\right) \\
& =1-f_{I F}\left(0, e_{8}, e_{7}+z\right)+f_{I F}\left(-1, e_{8}, e_{7}\right)-1 \\
& =e_{8}-e_{7}-z
\end{aligned}
$$

We have already chosen suitable values for $x$ and $y$ but $z$ is still free. Having worked with the 18-step collisions earlier, we realize that only suitable values for $z$ are $0,+1$ and -1 .

Equation 13: This equation is the easiest to satisfy. We need $\delta W_{12}=0$. But $\delta W_{12}=\delta f_{I F}^{11}(0,0, x)$. If the $f_{I F}$ function chooses its middle argument then we will have the desired. Hence we need to ensure $e_{11}=-1$. This can be done deterministically by choosing $W_{11}$ suitably.

Equation 8: This is a condition which needs to be satisfied. To get $\delta f_{M A J}^{7}(0,0, x)=0$, it is sufficient to ensure that $a_{7}=a_{6}$. This can be done deterministically by choosing $W_{7}$ suitably.

All the conditions are summarized in Table 3.

Table 3. Conditions put on the registers and differential path along with conditions yet to be satisfied.

\begin{tabular}{|c|c|c|cc|}
\hline 1 & $x=1, y=-1$ & 5 & $e_{8}-z-e_{7}=0$ & $(11)$ \\
\hline 2 & $a_{4}=a_{3}, a_{5}=-1$ & 6 & $\delta f_{M A J}^{6}(0, x, 0)=-z$ & $(6)$ \\
\hline 3 & $a_{7}=a_{6}$ & 7 & $-x=\delta f_{I F}^{8}(0, z, y)$ & $(10)$ \\
\hline 4 & $e_{9}=-1, e_{11}=-1$ & 8 & $\delta f_{I F}^{10}(0, x, 0)=-z$ & $(12)$ \\
\hline
\end{tabular}

We need to consider three choices for $z: 0,1$ and -1 . The middle arguments to the $\delta f_{M A J}^{6}$ function are $a_{5}+1$ and $a_{5}$, both of which have already been set to specific values 0 and -1 respectively (Cf. Condition 2). This causes difficulty in the satisfaction of Condition 6 in Table 3 for $z=1$. Hence we consider the other two values for $z$ now.

\subsection{When $z=0$}

This is the same 20-step differential path considered in [6]. We now attempt to satisfy conditions 5 to 8 in Table 3.

- Taking $a_{6}=a_{4}$ satisfies condition 6 . This can be done by suitably choosing $W_{6}$.

- Taking $e_{8}=e_{7}$ satisfies condition 5 . This can be done by suitably choosing $W_{8}$.

- Taking $e_{10}=0$ satisfies condition 8 . This can be done by suitably choosing $W_{10}$.

The only condition remaining now is Condition 7 which is $\delta f_{I F}^{8}(0,0,-1)=-1$. There is no message freedom left to satisfy this condition. In [6], this condition is let to be free and is satisfied with probability $1 / 3$ by random choices of messages. We now show that it is possible to satisfy even this condition deterministically.

It is clear that if we have $e_{8}=0$ then $f_{I F}$ will select its last argument which has a difference of -1 . Thus the output of $f_{I F}$ will be -1 as desired. But we have already chosen $W_{8}$ such that $e_{8}=e_{7}$. All the earlier message words starting from $W_{4}$ have also been used to satisfy some condition or the other. We now look at the calculation of $e_{7}$ :

$$
\begin{aligned}
e_{7} & =d_{6}+\Sigma_{1}\left(e_{6}\right)+f_{I F}\left(e_{6}, f_{6}, g_{6}\right)+h_{6}+K_{7}+W_{7} \\
& =d_{6}+a_{7}-\Sigma_{0}\left(a_{6}\right)-f_{M A J}\left(a_{6}, b_{6}, c_{6}\right) \\
& =a_{3}+a_{7}-\Sigma_{0}\left(a_{6}\right)-f_{M A J}\left(a_{6}, a_{5}, a_{4}\right) \\
& =a_{4}+a_{6}-\Sigma_{0}\left(a_{6}\right)-f_{M A J}\left(a_{6},-1, a_{4}\right)
\end{aligned}
$$


If we can ensure that $a_{6}=a_{4}=0$ then $e_{7}=e_{8}=0$ will be deterministic, which in turn will lead to a 20 -step collision with probability 1 . We used $W_{4}$ to get $a_{4}=a_{3}$ earlier. Now we choose the free word $W_{3}$ to get $a_{3}=0$. Rest of the conditions remain the same as in [6] and we get 20-step deterministic collisions for SHA-2. Examples of colliding message pairs for 20-step SHA-256 and SHA-512 are given in Section A. The set of conditions on the registers are given as Case 1 in Table 4.

Table 4. Conditions on the registers for 20-step deterministic collisions for SHA-2. Satisfaction of these conditions lead to 20-step collisions for SHA-2 with probability 1 . A condition on $a_{i}$ (or $e_{i}$ ) can be satisfied by suitable choice of $W_{i}$. The condition on $e_{7}$ in each case gets satisfied automatically when other conditions are met.

\begin{tabular}{|c|lll|}
\hline Case 1 & $x=1, y=-1$, & $z=0$ & \\
\hline 1 & $a_{3}=a_{4}=0$, & $a_{5}=-1$, & $a_{6}=a_{7}=0$ \\
\hline 2 & $e_{7}=e_{8}=0$, & $e_{10}=0$, & $e_{9}=e_{11}=-1$ \\
\hline Case $2-\mathrm{A}$ & $x=1, y=-1$, & $z=-1$ & \\
\hline 1 & $a_{3}=a_{4}=-1$, & $a_{5}=-1$, & $a_{6}=a_{7}=0$ \\
\hline 2 & $e_{7}=0, e_{8}=-1$, & $e_{9}=-1$, & $e_{10}=e_{11}=-1$ \\
\hline Case $2-\mathrm{B}$ & $x=1, y=-1$, & $z=-1$ & \\
\hline 1 & $a_{3}=a_{4}=0$, & $a_{5}=-1$, & $a_{6}=a_{7}=-1$ \\
\hline 2 & $e_{7}=1, e_{8}=0$, & $e_{9}=-1$, & $e_{10}=e_{11}=-1$ \\
\hline
\end{tabular}

\subsection{When $z=-1$}

Similar to the case $z=0$ above, we can determine conditions for 20-step collisions in SHA-2 and deterministically satisfy all the conditions. This time we get two sets of conditions. These are listed as Case 2-A and 2-B in Table 4. Note that this case gives rise to a new 20-step differential path for SHA-2. Colliding pairs of messages satisfying these conditions are given in Section A.

\section{Extending a Single Local Collision to Obtain 21-Step Collisions}

Using a single local collision to obtain 21-step collisions appears difficult because initial message words start repeating in the recursion of the message expansion this time. In [6], a single local collision spanning from Step 6 to Step 14 is used and a 21-step collision for SHA-256 is obtained probabilistically. Note that the earlier 20-step collisions had the local collision spanning from Step 5 to Step 13. This time the local collision has been slid down by one step. We first describe the method used in [6].

First 5 steps of message expansion for SHA-2 are:

$$
\begin{aligned}
& W_{16}=\underline{\sigma_{1}\left(W_{14}\right)+W_{9}}+\sigma_{0}\left(W_{1}\right)+W_{0} \\
& W_{17}=\sigma_{1}\left(W_{15}\right)+W_{10}+\sigma_{0}\left(W_{2}\right)+W_{1} \\
& W_{18}=\underline{\sigma_{1}\left(W_{16}\right)+W_{11}+\sigma_{0}\left(W_{3}\right)+W_{2}} \\
& W_{19}=\sigma_{1}\left(W_{17}\right)+W_{12}+\sigma_{0}\left(W_{4}\right)+W_{3} \\
& W_{20}=\underline{\sigma_{1}\left(W_{18}\right)}+W_{13}+\sigma_{0}\left(W_{5}\right)+W_{4}
\end{aligned}
$$

Since the chosen local collision has 4 consecutive zero message differentials within its span, we have $\delta W_{i}=0$ for $i \in\{10,11,12,13\}$. Further, this being the only local collision, messages outside the span of the local collision do not have any difference. Thus, we also have $\delta W_{i}=0$ for $i \in\{0,1,2,3,4,5,15\}$. Terms which may have non-zero differentials in the above equations are underlined.

All these zero differentials imply that if $\delta \sigma_{1}\left(W_{14}\right)+\delta W_{9}=0$ then the first 5 steps of the message expansion will not produce any difference, and we will have a 21-step collision. Since both $W_{14}$ and $W_{9}$ are random, it can be expected that they will cancel the differences in this manner. The probability 
for this cancellation to happen is estimated to be about $2^{-17.5}$ in [6]. Since their local collision has probability roughly $1 / 3$, the probability of the 21 -step collision is estimated to be approximately $2^{-19}$.

We use the same technique for our deterministic 20-step collisions and slide the single local collision one step to attempt a 21-step collision. We first observe that in having the 20-step collisions with probability 1 , we have lost some message freedom and consequently, $\delta W_{9}$ is no more random for two of the three cases described in Table 4. This happens for Case 1 and Case 2-B from this table. For proof of this claim, see Section B.

To use the 20-step collision described by Case 1 in Table 4, we need to relax some of the conditions there and obtain some randomness in $\delta W_{9}$. An example of such a relaxation is not to enforce $a_{3}=a_{4}=0$, rather only ensure $a_{3}=a_{4}$. This also causes relaxation on the condition on $e_{7}$, and the 20-step collision becomes probabilistic now. In fact, this is exactly the same 20-step collision described in [6]. The 21-step collision can now be found for this case as described in [6]. We describe an improvement to the search for messages satisfying $\delta \sigma_{1}\left(W_{14}\right)+\delta W_{9}=0$ a little later.

We note that the conditions in case 2 -B of Table 4 cannot be relaxed to obtain randomness in $\delta W_{9}$ and consequently this case can not be used for 21-step collisions. We also note that Case 2-A introduces randomness in $\delta W_{9}$ by default, so we do not need to relax any condition for this case. This is a good case for obtaining 21-step collisions, since it has probability 1 for all the steps other than the cancellation of $\delta W_{9}$ as described above. Next we describe our improved method of searching for suitable messages such that the difference in $W_{14}$ and $W_{9}$ cancels the difference in $W_{18}$.

\subsection{Obtaining messages satisfying $\delta \sigma_{1}\left(\delta W_{14}\right)+\delta W_{9}=0$}

We have that $\delta W_{14}=W_{14}^{\prime}-W_{14}=-1$. We expect $\delta W_{9}$ to be random. It is stated in [6] that by random choice of message words, the condition above can be satisfied with probability $2^{-17.5}$. This expectation seems to be based on the randomness of $\delta \sigma_{1}\left(W_{14}\right)$. We note that the difference of two $\sigma_{1}$ terms when their inputs differ by -1 is highly non-random.

The choices made in the local collision make the term $\delta W_{9}$ biased towards values which are small in magnitude. A rough idea of the distribution of $\delta W_{9}$ can be had from the following example: We ran the code for 21-step collisions of [6] $5 \times 10^{5}$ times and observed that only 174 times the value of $\delta W_{9}$ came out to be larger than 1000 in magnitude. Further, there were only 334 values larger than 500, 594 values larger than 300 and 1870 values larger than 100.

At the same time, $\sigma_{1}\left(W_{14}-1\right)-\sigma_{1}\left(W_{14}\right)$ is biased towards large magnitudes for random values of $W_{14}$. In fact, for a large number of points $p \in\{0,1\}^{32}$ there is no solution to the equation $\sigma_{1}\left(W_{14}-\right.$ $1)-\sigma_{1}\left(W_{14}\right)=-\delta W_{9}=p$. Interestingly, this equation does not have any solution for $W_{14}$ for even values of $p$. The distribution of the left hand side of this equation is so non-uniform that there are only 4 values of $\delta W_{9}$ in $\{-300,300\}$ for which a solution for $W_{14}$ exists. We list these 4 values of $\delta W_{9}$ and corresponding values of $W_{14}$ in Table 5 .

Table 5. Some solutions to the equation $\sigma_{1}\left(W_{14}-1\right)-\sigma_{1}\left(W_{14}\right)+\delta W_{9}=0$ for SHA-256.

\begin{tabular}{|c|c|l|}
\hline No. & $\delta W_{9}$ & $W_{14}$ \\
\hline 1 & 00000041 & $7 \mathrm{fc} 00000,80400000$ \\
\hline 2 & 00000101 & $\mathrm{~d} 5000000,81000000,7 \mathrm{f} 000000,2 \mathrm{~b} 000000$ \\
\hline 3 & $\mathrm{ffffff} 41$ & $4 \mathrm{c} 400000, \mathrm{~b} 3 \mathrm{c} 00000$ \\
\hline 4 & $\mathrm{ffffff} 01$ & $19000000,4 \mathrm{~d} 000000, \mathrm{~b} 3000000, \mathrm{e} 7000000$ \\
\hline
\end{tabular}

This analysis suggests that a specific suitable value of $\delta W_{9}$ should first be selected and then we should search for corresponding $W_{14}$. Even if this procedure is used, the probability of being able to get the correct $W_{14}$ is of the order of $2^{-32}$. This implies that the search in [6] is not over random messages, 
rather a pre-computed value of $W_{14}$ is used for a specific $\delta W_{9}$. From the colliding message pair given in [6], we observe that the value of $\delta W_{9}$ used is $f f f f f f 01$ and the corresponding $W_{14}$ is 19000000 . This particular choice of $\delta W_{9}$ occurs with probability $2^{-17.5}$ which corresponds to the estimate given in [6].

We use a speed-up in the search for the correct $W_{14}$. First we create a list of pairs $\left(\sigma_{1}(p)-\sigma_{1}(p-1), p\right)$ for all $p \in\{0,1\}^{32}$. We sort this list on the first element. While running the code for 21-step collision, we compute $\delta W_{9}$ and do a binary search over this list. If this value matches with the first element of a pair in the list, then we use the second element to define $W_{14}$. With this improvement, we obtain a 16 fold improvement to the probability of obtaining the correct $\delta W_{9}$. Since $W_{14}$ is pre-computed, the only probability is in getting the right difference $\delta W_{9}$.

We have extended two types of 20-step collisions to obtain 21-step collisions for SHA-256. One of the local collisions is the Case 1 of Table 4 with some conditions relaxed. As already mentioned, this is the Nikolić-Biryukov local collision [6] having probability $1 / 3$. For this case our method succeeds in finding correct $\delta W_{9}$ with probability roughly $2^{-13.5}$. Thus the overall probability of the 21-step SHA-256 collision is about $2^{-15}$.

The second 20-step collision we extend to 21 steps is described by Case 2-A of Table 4. For this case, we could find suitable $\delta W_{9}$ with probability roughly $2^{-17}$. Since the probability of the 20 -step collision is 1 in this case, we get the 21 -step collision with probability roughly $2^{-17}$.

\section{Conclusions}

In this paper we presented a generalized local collision for SHA-2. Using a single instance of this local collision, we obtained 18-step collisions with an arbitrary starting message difference. These collisions hold with probability 1 . We then presented two different differential paths for 20-step collisions in SHA-2 both of which hold with probability 1 . Finally, we improved on the search for 21-step collisions in SHA256 increasing the probability of success 16 fold. Apart from the colliding message pairs for different cases and different number of steps for SHA-256, we also show colliding message pairs for up to 20-step SHA-512 for the first time in the literature.

\section{Acknowledgements}

We would like to thank anonymous reviewers of ACISP 2008 for giving useful suggestions.

\section{References}

1. Florent Chabaud and Antoine Joux. Differential Collisions in SHA-0. In Hugo Krawczyk, editor, Advances in Cryptology - CRYPTO 1998, 18th Annual International Cryptology Conference, Santa Barbara, California, USA, August 23-27, 1998, Proceedings, volume 1462 of Lecture Notes in Computer Science, pages 56-71. Springer, 1998.

2. Henri Gilbert and Helena Handschuh. Security Analysis of SHA-256 and Sisters. In Mitsuru Matsui and Robert J. Zuccherato, editors, Selected Areas in Cryptography, 10th Annual International Workshop, SAC 2003, Ottawa, Canada, August 14-15, 2003, Revised Papers, volume 3006 of Lecture Notes in Computer Science, pages 175-193. Springer, 2003.

3. Philip Hawkes, Michael Paddon, and Gregory G. Rose. On Corrective Patterns for the SHA-2 Family. Cryptology eprint Archive, August 2004. Available at http://eprint.iacr.org/2004/207.

4. Florian Mendel, Norbert Pramstaller, Christian Rechberger, and Vincent Rijmen. Analysis of Step-Reduced SHA-256. In Matthew J. B. Robshaw, editor, Fast Software Encryption, 13th International Workshop, FSE 2006, Graz, Austria, March 15-17, 2006, Revised Selected Papers, volume 4047 of Lecture Notes in Computer Science, pages 126-143. Springer, 2006.

5. Florian Mendel, Norbert Pramstaller, Christian Rechberger, and Vincent Rijmen. Analysis of Step-Reduced SHA-256. Cryptology eprint Archive, March 2008. Available at http://eprint.iacr.org/2008/130.

6. Ivica Nikolić and Alex Biryukov. Collisions for Step-Reduced SHA-256. In Kaisa Nyberg, editor, Fast Software Encryption 2008, volume Pre-proceedings version of Lecture Notes in Computer Science, pages 1-16. Springer, 2008.

7. Somitra Kumar Sanadhya and Palash Sarkar. New Local Collisions for the SHA-2 Hash Family. In Kil-Hyun Nam and Gwangsoo Rhee, editors, Information Security and Cryptology - ICISC 2007, 10th International Conference, Seoul, Korea, November 29-30, 2007, Proceedings, volume 4817 of Lecture Notes in Computer Science, pages 193-205. Springer, 2007. 
8. Somitra Kumar Sanadhya and Palash Sarkar. Attacking Reduced Round SHA-256. In Steven Bellovin and Rosario Gennaro, editors, Applied Cryptography and Network Security - ACNS 2008, 6th International Conference, New York, NY, June 03-06, 2008, Proceedings, volume To appear of Lecture Notes in Computer Science. Springer, 2008.

9. Victor Shoup, editor. Advances in Cryptology - CRYPTO 2005: 25th Annual International Cryptology Conference, Santa Barbara, California, USA, August 14-18, 2005, Proceedings, volume 3621 of Lecture Notes in Computer Science. Springer, 2005.

10. Secure Hash Standard. Federal Information Processing Standard Publication 180-2. U.S. Department of Commerce, National Institute of Standards and Technology(NIST), 2002. Available at http://csrc.nist.gov/publications/ fips/fips180-2/fips180-2withchangenotice.pdf.

11. Xiaoyun Wang, Yiqun Lisa Yin, and Hongbo Yu. Finding Collisions in the Full SHA-1. In Shoup [9], pages 17-36.

12. Xiaoyun Wang and Hongbo Yu. How to Break MD5 and Other Hash Functions. In Ronald Cramer, editor, Advances in Cryptology - EUROCRYPT 2005, 24th Annual International Conference on the Theory and Applications of Cryptographic Techniques, Aarhus, Denmark, May 22-26, 2005, Proceedings, volume 3494 of Lecture Notes in Computer Science, pages 19-35. Springer, 2005.

13. Xiaoyun Wang, Hongbo Yu, and Yiqun Lisa Yin. Efficient Collision Search Attacks on SHA-0. In Shoup [9], pages $1-16$.

\section{A Colliding message pairs}

Table 6. Colliding message pair for 18-step SHA-256 with standard IV. These messages follow the differential path of Table 1 with $x=$ b875622d, $y=\mathrm{e} 4 \mathrm{bfa}$ ab5, $z=0$.

\begin{tabular}{|c|c|c|c|c|c|c|c|c|c|}
\hline \multirow{2}{*}{$\mathrm{W}_{1}$} & \multirow{2}{*}{$\begin{array}{c}0-7 \\
8-15\end{array}$} & $e 6 f$ & $58 f 290 f 9$ & $53 a c 42 f a$ & $3 a 7 c 9 e e 6$ & $30 \mathrm{dc} 2357$ & $2 \mathrm{ee} 1 \mathrm{~b} 785$ & Oabebaa2 & f61d8c82 \\
\hline & & $147 \mathrm{e} 048 \mathrm{~b}$ & $501 \mathrm{bc} 66 \mathrm{~b}$ & $75 a 3 d 802$ & $3 c 9 c a 879$ & $8 f 454627$ & $8 b 3 f f 382$ & $55 a 4 d e 5 a$ & a3e613ea \\
\hline \multirow[t]{2}{*}{$\mathrm{W}_{2}$} & $0-7$ & e6f590fc & $58 f 290 f 9$ & $53 a c 42 f a$ & f2f 20113 & $87 \mathrm{~b} 66 \mathrm{fa} 8$ & 77801baf & $57 \mathrm{~d} 16843$ & 9da87bd1 \\
\hline & $8-15$ & $9 \mathrm{~d} 408 \mathrm{abf}$ & $501 \mathrm{bc} 66 \mathrm{~b}$ & $75 a 3 d 802$ & $8427464 c$ & $8 f 454627$ & $8 b 3 f f 382$ & $55 a 4 d e 5 a$ & a3e613ea \\
\hline
\end{tabular}

Table 7. Colliding message pair for 18-step SHA-256 with standard IV. These messages follow the differential path of Table 1 with $x=60097 \mathrm{ffe}, y=$ a5dba93b, $z=x$.

\begin{tabular}{|c|c|c|c|c|c|c|c|c|c|}
\hline \multirow[t]{2}{*}{$\mathrm{W}_{1}$} & $0-7$ & $9868945 f$ & $43 e 023 b 2$ & $672 \mathrm{e} 208 \mathrm{~d}$ & d5c4df8c & 294d3db9 & a7bbabdc & $20 \mathrm{ff} 800 \mathrm{~b}$ & 76 bad5a7 \\
\hline & $8-15 \mid$ & $1 \mathrm{e} 09 \mathrm{c} 4 \mathrm{ef}$ & e778eba6 & 406 fc989 & of $0 f 6380$ & b91e9155 & $7965 e 503$ & $f 4 c 4 c 13 a$ & $57301 \mathrm{~b} 93$ \\
\hline \multirow[t]{2}{*}{$\mathrm{W}_{2}$} & $0-7$ & $9868945 f$ & $43 \mathrm{e} 023 \mathrm{~b} 2$ & $672 \mathrm{e} 208 \mathrm{~d}$ & $35 c e 5 f 8 a$ & d3a2bd52 & $63 f f 4094$ & |c0be1992 & $78 \mathrm{~b} 4 \mathrm{cf} 6 \mathrm{e}$ \\
\hline & $8-15 \mid$ & 3407 e934 & e778eba6 & 406 fc989 & af05e382 & b91e9155 & $7965 e 503$ & $f 4 c 4 c 13 a$ & b93 \\
\hline
\end{tabular}

Table 8. Colliding message pair for 18-step SHA-256 with standard IV. These messages follow the differential path of Table 1 with $x=$ ec1fe92d, $y=$ a01beee5, $z=-x=13 \mathrm{e} 016 \mathrm{~d} 3$.

\begin{tabular}{|c|c|c|c|c|c|c|c|c|c|}
\hline \multirow{2}{*}{$\mathrm{W}_{1}$} & $\overline{0-7}$ & eda26041 & 7 ea8c572 & $74155 \mathrm{~b} 82$ & $\mathrm{~d} 4 \mathrm{~d} 697 \mathrm{e} 9$ & $a 8 c 75 b 74$ & $c d c 3 d b a 6$ & $\overline{b 6 b c 5 d 2 f}$ & $2 \mathrm{~b} 2 \mathrm{f} c 241$ \\
\hline & $8-15$ & $51 \mathrm{~d} 8186 a$ & $416 \mathrm{~d} 969 f$ & $\overline{0 e b 5 c d 0 c}$ & $7044 f f 7 e$ & $0731645 f$ & $464 c 0913$ & $\mathrm{~d} 7 \mathrm{~d} 58642$ & $896 \mathrm{f} 7 \mathrm{bdb}$ \\
\hline \multirow[t]{2}{*}{$\overline{\mathrm{W}_{2}}$} & $0-7$ & eda26041 & 7 ea8c572 & $74155 \mathrm{~b} 82$ & $\operatorname{cof} 68116$ & $\mathrm{~d} 75 \mathrm{df} 145$ & $9 \mathrm{cc03075}$ & $7 f \mathrm{ff} 9 \mathrm{~d} 26$ & ef2fe209 \\
\hline & $8-15$ & 5678aad9 & $416 \mathrm{~d} 969 \mathrm{f}$ & Oeb5 & 84251651 & $0731645 f$ & $464 c 0913$ & d7d58642 & 8961 \\
\hline
\end{tabular}

\section{B Impossibility of some 21-step differential paths}

We now show that by one step sliding of a 20-step collision for Cases 1 and 2-B of Table 4, we cannot obtain a 21-step collision for SHA-2. 
Table 9. Colliding message pair for 20-step SHA-256 with standard IV. These messages follow the differential path of Table 1 with $x=1, y=-1, z=0$. These messages satisfy Case 1 of Table 4 .

\begin{tabular}{|c|c|c|c|c|c|c|c|c|c|}
\hline \multirow[t]{2}{*}{$\mathrm{W}_{1}$} & \multirow{2}{*}{$\begin{array}{c}0-7 \\
8-15\end{array}$} & $17 \mathrm{cf} 6 \mathrm{aff}$ & 89e9ba13 & c90b578d & b0db265f & ba7c84b0 & a24899eb & $980 \mathrm{f} 02 \mathrm{~b} 7$ & $627 \mathrm{ec} 4 \mathrm{ec}$ \\
\hline & & efaf $5 \mathrm{~d} 4 \mathrm{e}$ & $4 \mathrm{cb} 1 \mathrm{ae} 36$ & $157 \mathrm{~b} 67 \mathrm{~d} 7$ & $3 c d c 84 e 2$ & $d 9 d 4 c 9 a c$ & $0 c 32 f 8 c a$ & $5 a 262489$ & $86 f 0592 b$ \\
\hline $\mathrm{W}_{2}$ & $0-7$ & $17 \mathrm{cf} 6 \mathrm{aff}$ & $89 \mathrm{e} 9 \mathrm{ba} 13$ & c90b578d & b0db265f & $\mathrm{ba} 7 \mathrm{c} 84 \mathrm{~b} 0$ & a24899ec & 93 ef 0235 & $\mathrm{c} 56 \mathrm{e}$ \\
\hline & $8-15$ & efaf $5 d 4 d$ & $4 \mathrm{cb} 1 \mathrm{ae} 36$ & $157 \mathrm{~b} 67 \mathrm{~d} 7$ & $4 \mathrm{e} 2$ & d9d4c9ac & $0 c 32 f 8 c 9$ & $5 a 262489$ & $86 f 0592 b$ \\
\hline
\end{tabular}

Table 10. Colliding message pair for 20-step SHA-256 with standard IV. These messages follow the differential path of Table 1 with $x=1, y=-1, z=-1$. These messages satisfy Case 2-A of Table 4.

\begin{tabular}{|c|c|c|c|c|c|c|c|c|c|}
\hline \multirow[t]{2}{*}{$\mathrm{W}_{1}$} & $0-7$ & $\mathrm{a} 603 \mathrm{c} 44$ & 0 f 5 fdd 15 & $69 \mathrm{e} 8 \mathrm{c} 2 \mathrm{a} 4$ & $1754 c 271$ & 60518701 & feef6b5f & c7f50d13 & fdc492ca \\
\hline & $8-15$ & 53 & $d 4 c 9$ & $\mathrm{bf} 796 \mathrm{ac4}$ & af3823e & a24e8e62 & $8 c 8$ & fc4456f3 & $\mathrm{ae5}$ \\
\hline \multirow[t]{2}{*}{$\overline{\mathrm{W}_{2}}$} & $0-7$ & $5 a 603 c 44$ & $0 f 5 f d d 15$ & $69 \mathrm{e} 8 \mathrm{c} 2 \mathrm{a} 4$ & $1754 c 271$ & 60518701 & feef $6 \mathrm{~b} 60$ & d3d50e93 & f9a49248 \\
\hline & $8-15$ & \begin{tabular}{|c|}
$d 2326157$ \\
\end{tabular} & $d 4 c 9 d 37 f$ & bf796ac4 & aaf3823e & $a 24 e 8 e 62$ & $8 \mathrm{~d} 8898 \mathrm{c} 7$ & fc4456f3 & $8 d 557 a e 5$ \\
\hline
\end{tabular}

Table 11. Colliding message pair for 20-step SHA-256 with standard IV. These messages follow the differential path of Table 1 with $x=1, y=-1, z=-1$. These messages satisfy Case 2 -B of Table 4 .

\begin{tabular}{|c|c|c|c|c|c|c|c|c|c|}
\hline \multirow[t]{2}{*}{$\mathrm{W}_{1}$} & $0-7$ & $f 9 b$ & $4 \mathrm{e} 18 \mathrm{~d} 30 \mathrm{f}$ & $066 \mathrm{c} 47 \mathrm{~b} 9$ & $380 \mathrm{fb} 811$ & $364 \mathrm{c} 2 \mathrm{fb} 9$ & afac & $8 \mathrm{~d} 99$ & 80 \\
\hline & $8-15$ & $2 \mathrm{e} 182279$ & 92e6647c & $2263 \mathrm{df} 08$ & aaf3823e & 46 efda92 & $400 \mathrm{ed} 683$ & $56 \mathrm{bba} 6 \mathrm{ad}$ & c7133d81 \\
\hline \multirow[t]{2}{*}{$\mathrm{W}_{2}$} & $0-7$ & f9b685e2 & $4 \mathrm{e} 18 \mathrm{~d} 30 \mathrm{f}$ & $066 \mathrm{c} 47 \mathrm{~b} 9$ & $380 \mathrm{fb} 811$ & $364 \mathrm{c} 2 \mathrm{fb} 9$ & 085aafad & $917999 \mathrm{~b} 0$ & 1b332dff \\
\hline & $8-15$ & $323822 f 9$ & $92 e 6647 c$ & 2263df08 & aaf3823e & 46efda92 & 400ed682 & $56 \mathrm{bba} 6 \mathrm{ad}$ & c7133d81 \\
\hline
\end{tabular}

Table 12. Colliding message pair for 21-step SHA-256 with standard IV. These messages follow the differential path of Table 1 with $x=1, y=-1, z=0$. For these messages, $\delta W_{9}=$ ffffff 41 .

\begin{tabular}{|c|c|c|c|c|c|c|c|c|c|}
\hline$W_{1}$ & $0-7$ & f1497cd4 & 7fe4857c & df070eea & a035b751 & ece48886 & f42a8fc9 & fb1fe099 & 2052dc45 \\
& $8-15$ & $79 f 17 c 4 b$ & 8b1ee7ab & 85da1bdc & c07222ad & 3ccee34f & be164fd8 & b3c00000 & 571b5a2f \\
\hline $\mathrm{W}_{2}$ & $0-7$ & f1497cd4 & 7fe4857c & df070eea & a035b751 & ece48886 & f42a8fc9 & fb1fe09a & e233a2c1 \\
& $8-15$ & $75 d 17 a d d$ & 8b1ee6ec & 85da1bdc & c07222ad & 3ccee34f & be164fd8 & b3bfffff & 571b5a2f \\
\hline
\end{tabular}

Table 13. Colliding message pair for 21-step SHA-256 with standard IV. These messages follow the differential path of Table 1 with $x=1, y=-1, z=-1$. For these messages $\delta W_{9}=$ ffffe 191 .

\begin{tabular}{|c|c|c|c|c|c|c|c|c|c|}
\hline$W_{1}$ & $0-7$ & $4158 e c c 7$ & $3 a 3 f f e 61$ & ba7149f0 & ed452440 & 4d9ab924 & f016459f & 22f5578c & c56333c1 \\
& $8-15$ & ff1941ff & 19b8055b & fb2876ba & ca4d6044 & 8d41a28d & 8194372b & 7e100000 & 5240bb72 \\
\hline $\mathrm{W}_{2}$ & $0-7$ & $4158 e c c 7$ & 3a3ffe61 & ba7149f0 & ed452440 & 4d9ab924 & f016459f & 22f5578d & c1433241 \\
& $8-15$ & fb39427d & 19b7e6ec & fb2876ba & ca4d6044 & 8d41a28d & $8194372 b$ & 7 e0fffff & $5240 b b 72$ \\
\hline
\end{tabular}

Table 14. Colliding message pair for 18-step SHA-512 with standard IV. These messages follow the differential path of Table 1 with $x=373 \mathrm{c5915a7e8cd1a,} y=$ bac8b5823e5656cb, $z=0$.

\begin{tabular}{|c|c|l|l|l|l|}
\hline $\mathrm{W}_{1}$ & $0-3$ & eb1eb59ecf0b3342 & e205af0b51f81569 & 62759b3c1cbbfb60 & d94c8594e1468081 \\
\cline { 2 - 6 } & $4-7$ & 21c555e5eb4a74ff & 466534f9e5c4dd20 & 2c55b4e93bd76391 & 0dffe3bf30abcb91 \\
\cline { 3 - 6 } & $8-11$ & f2051be933e8762d & 57417ceddcf050ed & a7116f111de85809 & 5ed73acd8290c14c \\
\cline { 3 - 7 } & $12-15$ & d60a9f4bbcadc128 & 54ea8bb9f46b36ef & 3ac446634c581411 & cbf82d9f9493f84d \\
\hline $\mathrm{W}_{2}$ & $0-3$ & eb1eb59ecf0b3342 & e205af0b51f81569 & 62759b3c1cbbfb60 & 1088deaa892f4d9b \\
\cline { 2 - 6 } & $4-7$ & 537807237bcf8a95 & 1b8ab570b1112066 & 74a8eaf30dcdac7e & 1e2394a74a7a9b86 \\
\cline { 2 - 6 } & $8-11$ & aa49e4d0d4cfdbc7 & 57417ceddcf050ed & a7116f111de85809 & 279ae1b7daa7f432 \\
\cline { 2 - 6 } & $12-15$ & d60a9f4bbcadc128 & 54ea8bb9f46b36ef & 3ac446634c581411 & cbf82d9f9493f84d \\
\hline
\end{tabular}


Table 15. Colliding message pair for 18-step SHA-512 with standard IV. These messages follow the differential path of Table 1 with $x=800000000000000, y=7$ ffffff7df000000, $z=x$.

\begin{tabular}{|c|c|c|c|c|c|}
\hline \multirow[t]{4}{*}{$\mathrm{W}_{1}$} & $0-3$ & 4 d231bd0aee1913 & 988d1c29544b4e23 & $77641612867 \mathrm{aeOba}$ & c3b0ce9aee99e947 \\
\hline & $4-7$ & $4 a 65130318 d c c 860$ & $2 \mathrm{ffd} 17 \mathrm{efc9d} 7826 \mathrm{~d}$ & $8773 \mathrm{e} 9 \mathrm{f} 2 \mathrm{c} 175 \mathrm{c} 1 \mathrm{c7}$ & d8dcc93460a556ba \\
\hline & $8-11$ & f0f055a & e586 & 7476 & 2065 \\
\hline & $12-15$ & f97c79 & a631aa6ec & $9 \mathrm{c000af74}$ & $8 a 32144 b f d 97630 e$ \\
\hline \multirow[t]{4}{*}{$\mathrm{W}_{2}$} & $\overline{0-3}$ & $\mathrm{~d} 5 \mathrm{~d} 231$ & 988d1c29544 & 77641 & $43 \mathrm{~b} 0 \mathrm{ce} 9$ \\
\hline & $4-7$ & $4 a 62 f 2 f$ af $79 c c 860$ & b3ceb7df69bee62d & $877609 \mathrm{f} 2 \mathrm{a} 2 \mathrm{~b} 5$ & $40 \mathrm{a} 556 \mathrm{ba}$ \\
\hline & $8-11$ & 70ee75ad82390591 & $6586 \mathrm{e} 628 \mathrm{eca} 6 \mathrm{fdaa}$ & 74763eadbd1b619b & e3faa21560edc065 \\
\hline & $2-1$ & c799fa4a01d & 119e52a631aa6ec & $6 \mathrm{e} 76 \mathrm{e} 09 \mathrm{c} 000 \mathrm{af} 74$ & a32144bfd97 \\
\hline
\end{tabular}

Table 16. Colliding message pair for 18-step SHA-512 with standard IV. These messages follow the differential path of Table 1 with $x=$ c0145fc22e2f8106, $y=70 \mathrm{df} 70 \mathrm{~d} 99098 \mathrm{ebeb}, z=-x=3$ feba03dd1d07efa.

\begin{tabular}{|c|c|c|c|c|c|}
\hline \multirow[t]{4}{*}{$\widehat{\mathrm{W}_{1}}$} & $\overline{0-3}$ & 49447 f9cd22cbc1 & b56e3ca4d7d16a57 & 5bd5d12d24969ab4 & 0e2ea85d485ad0f9 \\
\hline & $4-7$ & $87 a f b a c 32285 a 4 a 7$ & 69bf 436266be288e & 46aa45bd104ef93c & 370586b96422ce9b \\
\hline & $8-11$ & d7534fa56ee15811 & $423 \mathrm{~b} 6$ & $91 \mathrm{e} 21$ & $d 4 \mathrm{~b} 4$ \\
\hline & $12-15$ & $06 a 12448353 c 4575$ & $358 \mathrm{db}$ & $5 d 1794 c 82$ & If $23262776 b 4$ \\
\hline \multirow[t]{4}{*}{$\mathrm{W}_{2}$} & $0-3$ & $9 \mathrm{~cd} 22 \mathrm{cbc} 1$ & $\mathrm{~b} 56 \mathrm{e} 3$ & $5 \mathrm{bd5}$ & $\mathrm{ce} 43$ \\
\hline & $4-7$ & e4ac8281951462fe & $9 \mathrm{~b} 6 \mathrm{cec}$ & dbcd070889 & $0 \mathrm{e} 21 \mathrm{~b} 1394492621 \mathrm{~b}$ \\
\hline & $8-11$ & $9 \mathrm{a} 5 \mathrm{eb} 6 \mathrm{e} 1 \mathrm{de} 5 \mathrm{fd} 1 \mathrm{a} 0$ & $423 \mathrm{~b} 664 \mathrm{e} 4392 \mathrm{c} 00 \mathrm{e}$ & 5013 & 4954b440c65253ae \\
\hline & $12-15$ & a12448353c4575 & $358 d b 4301 a 231 c 4 c$ & $5 d 1794 c 82015 a 66$ & $1464 f 23262776 b 4$ \\
\hline
\end{tabular}

Table 17. Colliding message pair for 20-step SHA-512 with standard IV. These messages follow the differential path of Table 1 with $x=1, y=-1, z=0$. These messages satisfy Case 1 of Table 4 .

\begin{tabular}{|c|c|c|c|c|c|}
\hline \multirow[t]{4}{*}{$\mathrm{W}_{1}$} & $0-3$ & 058ceb9a1077eb2 & $a 4 c f 55 c 2 b 1 b b 8 f c e$ & $784193965385 f f 3 b$ & $7463839 \mathrm{e} 2 \mathrm{fe} 1 \mathrm{~d} 369$ \\
\hline & $4-7$ & $88168 \mathrm{bd} 7 f 18 \mathrm{e} 72 \mathrm{a} 7$ & 2c4bba75ff7d74e6 & $2 \mathrm{aebc8365586a02d}$ & c3506e0db562134a \\
\hline & $8-11$ & $73 \mathrm{a} 7$ & $176 c$ & $\mathrm{ce} 3 \mathrm{~b}$ & $\overline{08 a c}$ \\
\hline & $2-15$ & f644b8df 45 fbe4d8 & $\mathrm{bb} 87 \mathrm{a} 43 \mathrm{dc0674b95}$ & 61d9c1b117244b44 & e2264ccbf7bf427e \\
\hline \multirow[t]{4}{*}{$\mathrm{W}_{2}$} & $\overline{0-3}$ & $6058 c \epsilon$ & $a 4 c f 55 c$ & 784 & 746383 \\
\hline & $4-7$ & $88168 \mathrm{bd} 7 \mathrm{f} 18 \mathrm{e} 72 \mathrm{a} 7$ & $2 \mathrm{c} 4 \mathrm{bba} 7$ & 2af008365506a02d & c34c2e0db5e2134a \\
\hline & $8-11$ & 73a70156e11e07c1 & $9947 f 674 a 891 d 76 c$ & 1023901ef5eace3b & b258c2dde4e508ac \\
\hline & $12-15$ & $644 \mathrm{~b} 8 \mathrm{df} 45 \mathrm{fbe} 4 \mathrm{~d} 8$ & ob87a43dc067 & d9c1b1 & \\
\hline
\end{tabular}

Table 18. Colliding message pair for 20-step SHA-512 with standard IV. These messages follow the differential path of Table 1 with $x=1, y=-1, z=-1$. These messages satisfy Case 2-A of Table 4 .

\begin{tabular}{|c|c|c|c|c|c|}
\hline $\mathrm{W}_{1}$ & $0-3$ & 1c99041525eeeeb3 & 7dfc74f74bab1a89 & aaca442cddb37351 & 21d1684a782a5b87 \\
\cline { 3 - 7 } & $4-7$ & 3d374aed94c9d766 & 296c28f080eced7a & 62f73e6df90ce266 & d4c85286272c52c1 \\
\cline { 3 - 7 } & $8-11$ & e2d8e832fb623115 & 5c43e3fc9bee94c3 & 5ef6f726192a4213 & aaf3823c2a004b1f \\
\hline & $12-15$ & fa18ffe92868d117 & 8584328bd3146ed0 & c3ce87104858e6cb & 6dc9cd6519344c6a \\
\hline$W_{2}$ & $0-3$ & 1c99041525eeeeb3 & 7dfc74f74bab1a89 & aaca442cddb37351 & 21d1684a782a5b87 \\
\cline { 3 - 6 } & $4-7$ & 3d374aed94c9d766 & 296c28f080eced7b & 62fafe6df88ce264 & d4cc928628ac52c0 \\
\cline { 3 - 6 } & $8-11$ & f73a261982122135 & 5c43e3fc9bee94c3 & 5ef6f726192a4213 & aaf3823c2a004b1f \\
\hline & $12-15$ & fa18ffe92868d117 & 8584328bd3146ecf & c3ce87104858e6cb & 6dc9cd6519344c6a \\
\hline
\end{tabular}


Table 19. Colliding message pair for 20-step SHA-512 with standard IV. These messages follow the differential path of Table 1 with $x=1, y=-1, z=-1$. These messages satisfy Case 2-B of Table 4.

\begin{tabular}{|c|c|c|c|c|c|}
\hline \multirow[t]{4}{*}{$\mathrm{W}_{1}$} & $0-3$ & $4 \mathrm{~cd} 8$ & & & \\
\hline & $4-7$ & $71 \mathrm{ec} 2 \mathrm{db}$ & d1a5 & $43 \mathrm{e} 6$ & $3 a c 1$ \\
\hline & 8 & $03 c f$ & $17 \mathrm{e} 2 \mathrm{a}$ & $\operatorname{de} 50$ & tb1f \\
\hline & $12-15$ & f3fd487aea68fbd9 & fcf6a431bae731ff & aba4536a50179e3d & $837 \mathrm{c} 2 \mathrm{afdff} 067 \mathrm{~b} 28$ \\
\hline \multirow[t]{4}{*}{$\mathrm{W}_{2}$} & $0-$ & $7 f 446 c 83$ & $f e 2 f$ & $368 \mathrm{~b}$ & 2c24db098? \\
\hline & $4-7$ & 71ec2db073b48f6a & 4c95a6faaa6dd1a6 & $1 \mathrm{f} 1 \mathrm{e} 485 \mathrm{~d} 9 \mathrm{f} 1643 \mathrm{e} 7$ & 3abe31ef5f7382ff \\
\hline & $8-11$ & $03 \mathrm{~d} 35 \mathrm{~b} 75851 \mathrm{~b} 5221$ & $1 \mathrm{~d} 5 \mathrm{c} 1436 \mathrm{e} 6417 \mathrm{e} 2 \mathrm{a}$ & 1b619cf7e4dfde50 & $a a f 3823 c 2 a 004 b 1 f$ \\
\hline & $12-15$ & f 3 fd 487 aea 68 fbd9 & fcf6a431bae731fe & a4536a50179e3d & afdff067b28 \\
\hline
\end{tabular}

First note that the cases described in Table 4 are for a local collision spanning from Steps 5 to 13. Now that we have shifted the local collision by one step to span it from Step 6 to Step 14, all the conditions of Table 4 also need to be shifted by one index. Hence a condition on $a_{i}$ in this table will become a condition on $a_{i+1}$ for our present case.

\section{B.1 Case 1: $(x=1, y=-1, z=0)$ :}

We have $a_{4}=a_{5}=a_{7}=a_{8}=0, a_{6}=-1, e_{8}=e_{9}=e_{11}=0, e_{10}=e_{12}=-1$. From (9), $\delta W_{9}=$ $-\delta f_{I F}^{8}(0,-1,1)-\delta \Sigma_{1}\left(e_{8}\right)$. Simplifying this we get:

$$
\begin{aligned}
\delta W_{9} & =f_{I F}\left(e_{8}, e_{7}, e_{6}\right)-f_{I F}\left(e_{8}, e_{7}-1, e_{6}+1\right)+\Sigma_{1}\left(e_{8}\right)-\Sigma_{1}\left(e_{8}\right) \\
& =f_{I F}\left(0, e_{7}, e_{6}\right)-f_{I F}\left(0, e_{7}-1, e_{6}+1\right)+\Sigma_{1}(0)-\Sigma_{1}(0) \\
& =e_{6}-\left(e_{6}+1\right)=-1
\end{aligned}
$$

We now need a pair of message words $W_{14}$ and $W_{14}^{\prime}=W_{14}-1$ such that $\delta W_{14}=-\delta W_{9}=1$. We note that there does not exist any 32 -bit word $W_{14}$ which can satisfy this condition.

B.2 Case 2-B: $(x=1, y=-1, z=-1)$ :

We have $a_{4}=a_{5}=0, a_{6}=a_{7}=a_{8}=-1, e_{8}=1, e_{9}=0, e_{10}=e_{11}=e_{12}=-1$. From (9), $\delta W_{9}=-\delta f_{I F}^{8}(-1,-1,1)-\delta \Sigma_{1}\left(e_{8}\right)$. Simplifying this we get:

$$
\begin{aligned}
\delta W_{9} & =f_{I F}\left(e_{8}, e_{7}, e_{6}\right)-f_{I F}\left(e_{8}-1, e_{7}-1, e_{6}+1\right)+\Sigma_{1}\left(e_{8}\right)-\Sigma_{1}\left(e_{8}-1\right) \\
& =f_{I F}\left(1, e_{7}, e_{6}\right)-f_{I F}\left(0, e_{7}-1, e_{6}+1\right)+\Sigma_{1}(1)-\Sigma_{1}(0) \\
& =f_{I F}\left(1, e_{7}, e_{6}\right)-f_{I F}\left(0, e_{7}-1, e_{6}+1\right)+\Sigma_{1}(1)
\end{aligned}
$$

The $f_{I F}$ function selects its output bit from either its second or third argument. Since the first arguments of the two $f_{I F}$ terms differ only at the lowest bit, the output from the difference of the two $f_{I F}$ terms can only be +1 or 0 or -1 . The last term $\Sigma_{1}(1)$ is a constant quantity. We now need a pair of message words $W_{14}$ and $W_{14}^{\prime}=W_{14}-1$ such that $\delta W_{14}=-\delta W_{9}$. We note that there does not exist any 32 -bit word $W_{14}$ which can satisfy this condition. 